\title{
The Twente Photoacoustic Mammoscope 2: 3D vascular network visualization
}

\author{
Sjoukje M. Schoustra*,a, Roeland Huijink ${ }^{\mathrm{b}}$, Laurens Alink ${ }^{\mathrm{b}}$, Tim J. P. M. op 't Root ${ }^{\mathrm{b}}$, Daan \\ Sprünken $^{\mathrm{b}}$, Daniele Piras ${ }^{\mathrm{a}}$, Wouter F. Muller Kobold ${ }^{\mathrm{b}}$, Caroline A. H. Klazen ${ }^{\mathrm{c}}$, Margreet C. van der \\ Schaaf $^{\mathrm{c}}$, Frank M. van den $\mathrm{Engh}^{\mathrm{c}}$, Wiendelt Steenbergen ${ }^{\mathrm{a}} \&$ Srirang Manohar $^{\mathrm{a}}$ \\ ${ }^{\mathrm{a} B i o m e d i c a l ~ P h o t o n i c ~ I m a g i n g ~ G r o u p, ~ T e c h n i c a l ~ M e d i c a l ~ C e n t r e, ~ U n i v e r s i t y ~ o f ~ T w e n t e, ~ P O ~ B o x ~}$ \\ 217, 7500 AE Enschede, The Netherlands; \\ ${ }^{\mathrm{b}}$ PA Imaging R\&S B.V., De Veldmaat 10, Bldg. 46 / HTF-SEPA HI.222-224, 7522 NM Enschede, \\ The Netherlands; ${ }^{\circ}$ Center for Breast Care, Medisch Spectrum Twente, PO Box 50000, 7500 KA \\ Enschede, The Netherlands
}

\begin{abstract}
We present the Twente Photoacoustic Mammoscope 2 (PAM 2) based on a 3D tomographic geometry. A functional optical contrast map of breast vascularization can be obtained in a noninvasive, radiation-free and painless manner. A woman lies prone on a bed with one breast pendant in an imaging tank with water, where 12 curved ultrasound arrays are mounted. Each array extends from chest wall towards the nipple following the contour of the pendant breast, and carries 32 detector elements. The detectors' center frequency is $1 \mathrm{MHz}$. The breast is illuminated from multiple directions: the ventral side of the breast from the bottom and the areas close to the chest wall from the sides. The excitation wavelengths are $755 \mathrm{~nm}$ and $1064 \mathrm{~nm}$. By rotating the imaging tank in between measurements, multiple projections can be obtained, providing a 3D image of the breast after reconstruction by means of a filtered backprojection. So far, breasts of healthy volunteers were imaged. Three-dimensional images of the breast contour, the nipple and blood vessel networks within the breast could be observed with high contrast and unprecedented detail.
\end{abstract}

Keywords: photoacoustic imaging, tomography, breast cancer, breast imaging, breast vascularization

\section{INTRODUCTION}

Breast cancer is the most frequently diagnosed cancer type, resulting in the highest number of cancer deaths among women worldwide ${ }^{1}$. For detection, diagnosis, treatment and follow-up of breast cancer, imaging techniques are of great importance ${ }^{2}$. The use of photoacoustics (PA) for breast imaging is being investigated around the world, in various imaging geometries and for various applications within the area of breast cancer care. The use of handheld photoacoustic probes for breast cancer diagnosis is investigated by multiple research groups, mostly combined with conventional ultrasound imaging ${ }^{3-7}$. Other research groups are working in the field of PA tomographic breast imaging, where a woman lies prone on a bed with one breast inside an imaging chamber. For example, the Kyoto-Canon system has a hemispherical detector $\operatorname{array}^{8}$ and uses a spiral scanning pattern to image the breast. They recently added the acquisition of ultrasonic B-mode images to their system ${ }^{9}$. The group of Oraevsky ${ }^{10}$ also have a hemispherical array where they coregister anatomical US images with functional PA images in their LOUISA-3D system. Further, Lin et al. ${ }^{11}$ recently showed a ring array of detectors which scans the pendant breast along the elevational axis within a single-breath-hold. We have presented the PAM 1 system $^{12}$, where a woman also lay prone on a bed and one breast was imaged. It employed a planar geometry in forward mode, where the breast was illuminated from the cranial side and photoacoustic signals were detected from the caudal side with a 2D ultrasound detector array. With the PAM 1 system, we have shown visualization of breast malignancies in vivo ${ }^{12-16}$. Here we present the Twente Photoacoustic Mammoscope 2 (PAM 2) based on a 3D tomographic geometry, and demonstrate its capability on the breast of a healthy volunteer.

*Corresponding author: s.m.schoustra@utwente.nl

Photons Plus Ultrasound: Imaging and Sensing 2019, edited by Alexander A. Oraevsky, Lihong V. Wang, Proc. of SPIE Vol. 10878, 1087813 - @ 2019 SPIE · CCC code: 1605-7422/19/\$18 - doi: 10.1117/12.2507448 


\section{MATERIALS AND METHODS}

\subsection{PAM 2 system}

The ultrasound detector system consists of 384 single elements, divided over 12 arrays (Imasonic SAS, Voray sur l'Ognon, France). One array consists of 32 piezocomposite elements, placed on an arc with a radius of $120 \mathrm{~mm}$. The 12 arrays were placed inside the imaging tank, following the curvature of the pendant breast. The detectors' center frequency is $1 \mathrm{MHz}$.

The PAM 2 system employs an Nd:YAG $(1064 \mathrm{~nm})$ and Alexandrite $(755 \mathrm{~nm})$ combined Q-switched laser to illuminate the breast (Qcombo, Quanta System, Milan, Italy). The breast is illuminated with a large diverging laser beam to the nipple side of the breast (called bottom illumination), but also by discrete illumination via 9 optical fiber bundles (called side illumination). The fiber bundles are directed slightly upward to access areas close to the chest wall and also contain a diverging lens.

During a measurement, a subjects lies prone on a bed with one breast through an aperture (diameter $21 \mathrm{~cm}$ ), pendant in the PAM 2 double-walled imaging tank (outer diameter $40.5 \mathrm{~cm}$, inner diameter $35.5 \mathrm{~cm}$ ). Water inside the tank is warmed up by circulating externally heated water inside the double wall. The configuration of fiber bundles and acoustic detector arrays inside the imaging tank is depicted in Figure 1. The bottom illumination beam is directed upwards through a window in the tank bottom. The imaging tank rotates in steps around the pendant breast, obtaining multiple projections. The number of projection angles and the number of laser pulses of which an average signal is derived per projection can be set for each experiment.
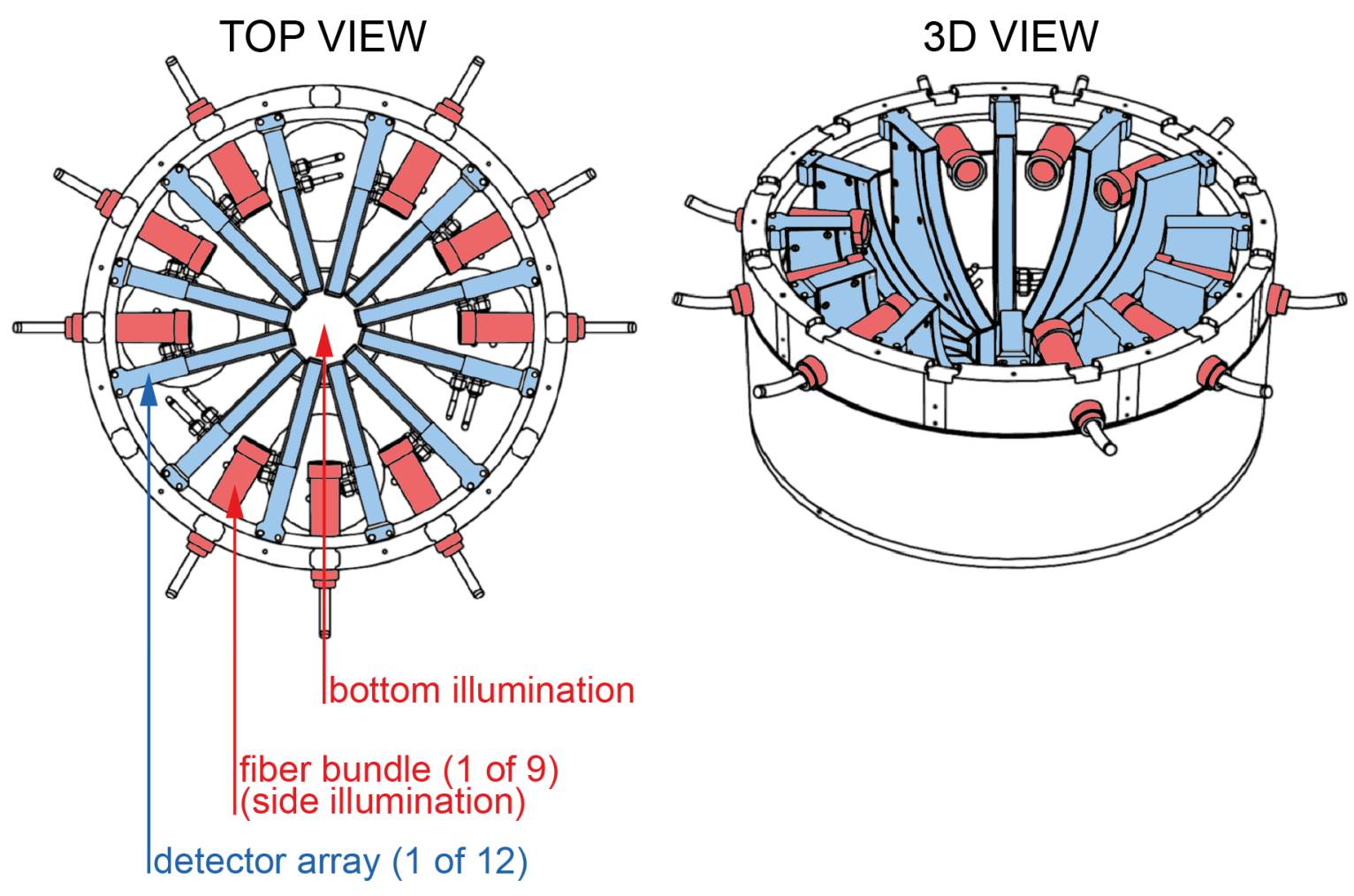

Figure 1. Top view drawing of the imaging tank. Red depicts illumination, blue depicts detection.

A waterproof stainless steel case encloses a detector array and its integrated preamp (PA Imaging R\&D B.V., Enschede, The Netherlands). Photoacoustic data is acquired through a 12 times 32 channels analog to digital converter (ADC) as part of the data acquisition (DAQ) system (PA Imaging R\&D B.V., Enschede, The Netherlands). Sampling is done with 
a frequency of $25 \mathrm{MHz}$ and a depth of 14 bits. For the reconstruction, a filtered backprojection based on the algorithm of Haltmeier et $a l .{ }^{17}$ was implemented. The system was comprehensively characterized for detector response and resolution using test-objects. Deconvolution of the measured signals before reconstuction is performed to account for the average frequency response of the detectors. Relative sensitivities of single detector elements are employed to scale the measured signals before reconstruction.

Instrument safety and hygiene aspects were studied in detail. Laser fluence on the skin is kept below the safety limit of $20 \mathrm{~mJ} / \mathrm{cm}^{2}$ at all times (NEN-EN-IEC 60825-1:2014). Safety goggles for eye protection are worn by the subject and operator(s) whenever the laser is emitting.

\subsection{Measurement protocol}

We will present typical images, obtained with our PAM 2 system at an excitation wavelength of $755 \mathrm{~nm}$, to show the performance and possibilities of the system in imaging the breast. Measurements were performed on a 29 year old Caucasian healthy volunteer (random number 154 was assigned). The procedure was explained and informed consent was obtained according to a protocol approved by the regional Medical Research and Ethics Committee (METC Twente), and the study has been registered in the Netherlands National Trial Register (NTR) (TC 6508).

The bottom illumination beam accounted for $67 \%$ of delivered light energy, the remaining 33\% was spread over the 9 side illumination fiber bundles. During all measurements, the temperature of the water bath in which the breast was pendant was approximately $26^{\circ} \mathrm{C}$. Tomographic scans were obtained. A measurement with 21 projection angles and 100 laser pulses per average was obtained. In between two consecutive projections, the imaging tank was rotated $3^{\circ}$. Imaging one breast with this measurement protocol took 4 minutes.

Reconstructed images (grid size $=0.4 \mathrm{~mm}$ ) are presented as maximum intensity projections (MIPs) in three planes (coronal, sagittal and transverse). Negative reconstructed values were set to zero. Images are also presented in the form of color-coded local maximum intensity projections (LMIPs) ${ }^{18}$. In an LMIP, the first pixel above a set threshold is plotted (compared to the maximum value pixel in MIP). We color-coded our LMIP images, where white means the pixel is close to the observer, orange means it is further away.

In the three MIP views, horizontal and vertical lines are indicating coordinates where slices through the volume were obtained. These slices are plotted separately as MIPs of a sub-volume with a thickness of $1.5 \mathrm{~mm}$. A video of the reconstructed $3 \mathrm{D}$ volume of the measurement was also created and is attached.

\section{RESULTS}

Figure 2a-c show color-coded LMIPs from images obtained of the left breast of healthy volunteer \#154, illuminated with $755 \mathrm{~nm}$. An illustration indicates the orientation of the breast in the various projections. An LMIP in the coronal (XY) plane, the sagittal (XZ) plane and the transverse plane (YZ) are shown. We clearly see a vascular network as well as the nipple and the breast contour (the latter in the sagittal and transverse views). The color-coding gives a sense of depth to the images.

Figure 3a-c show MIPs from the same measurement. The lines indicate the coordinates at which slices through the 3D volume were obtained and plotted in Figure 3d-e. For example, the vertical line in Figure 3a results in a transverse (YZ) view (Figure 2e) at the corresponding $\mathrm{x}$-coordinate. At the cross-section of the lines, a green marker was placed. This marker indicates the deepest point to which a certain vessel could be traced. This vessels is more clearly visible in the slices (Figure 3d-e) than in the MIPs (Figure 3a-c). The depth of this green marker was estimated to be at $20 \mathrm{~mm}$ with respect to the skin surface.

Video 1 shows the reconstructed volume rotating, providing a more intuitive interpretation of the volume compared to the 2D MIP and LMIP images. The volume is oriented such that the breast is in a supine position. We see the nipple clearly, and also the outer layer of breast tissue with vascularization. It can be seen that not the entire breast was imaged. This is also apparent in the transverse views (Figure $2 \mathrm{c}$ and Figure $3 \mathrm{c}$ and $3 \mathrm{e}$ ). The part caudal of the nipple extending to the chest wall is not visible. 


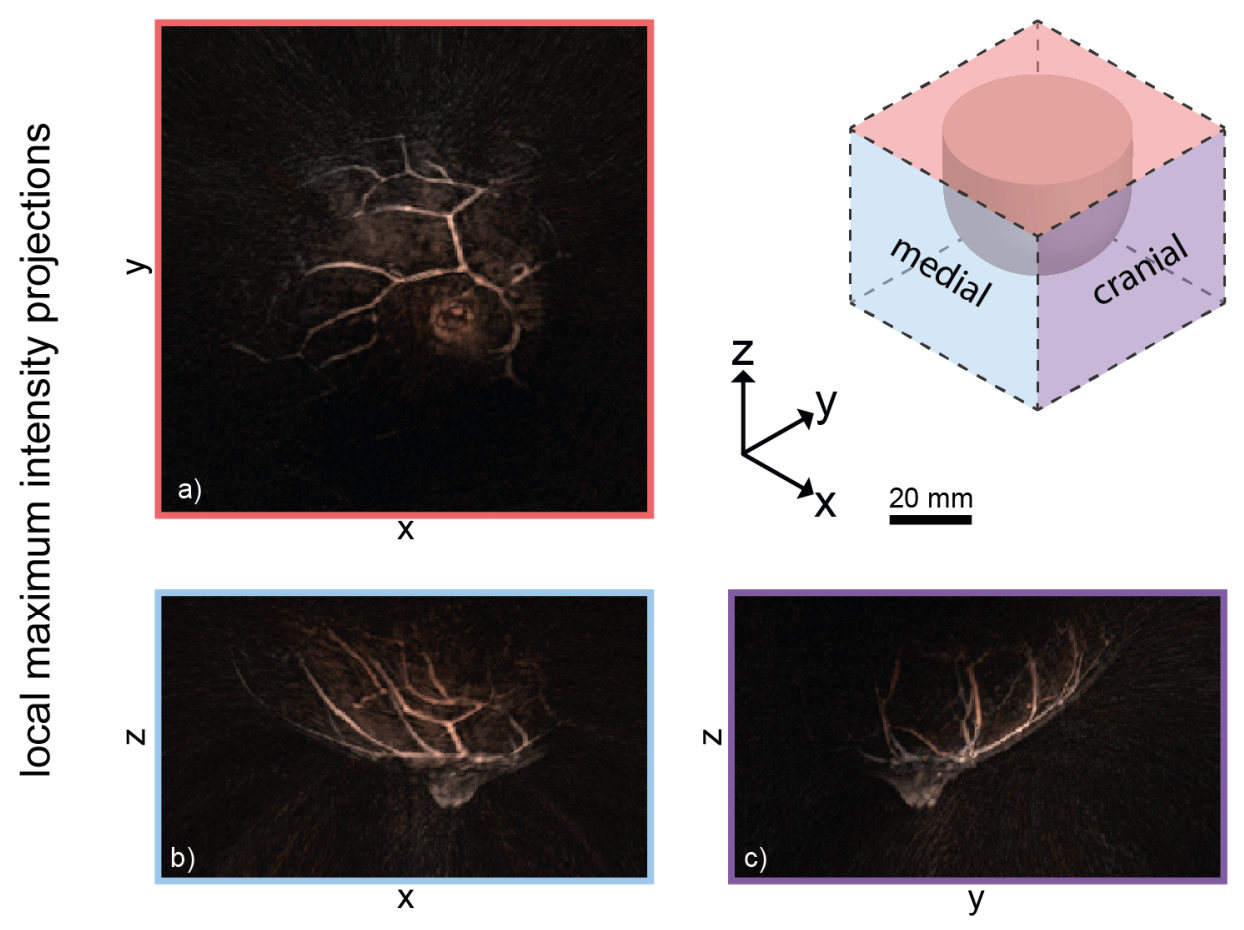

Figure 2. Reconstructed color-coded LMIPs of the left breast of healthy volunteer \#154. a) Coronal (XY view). b) Sagittal (XZ) view). c) Transverse (YZ) view.

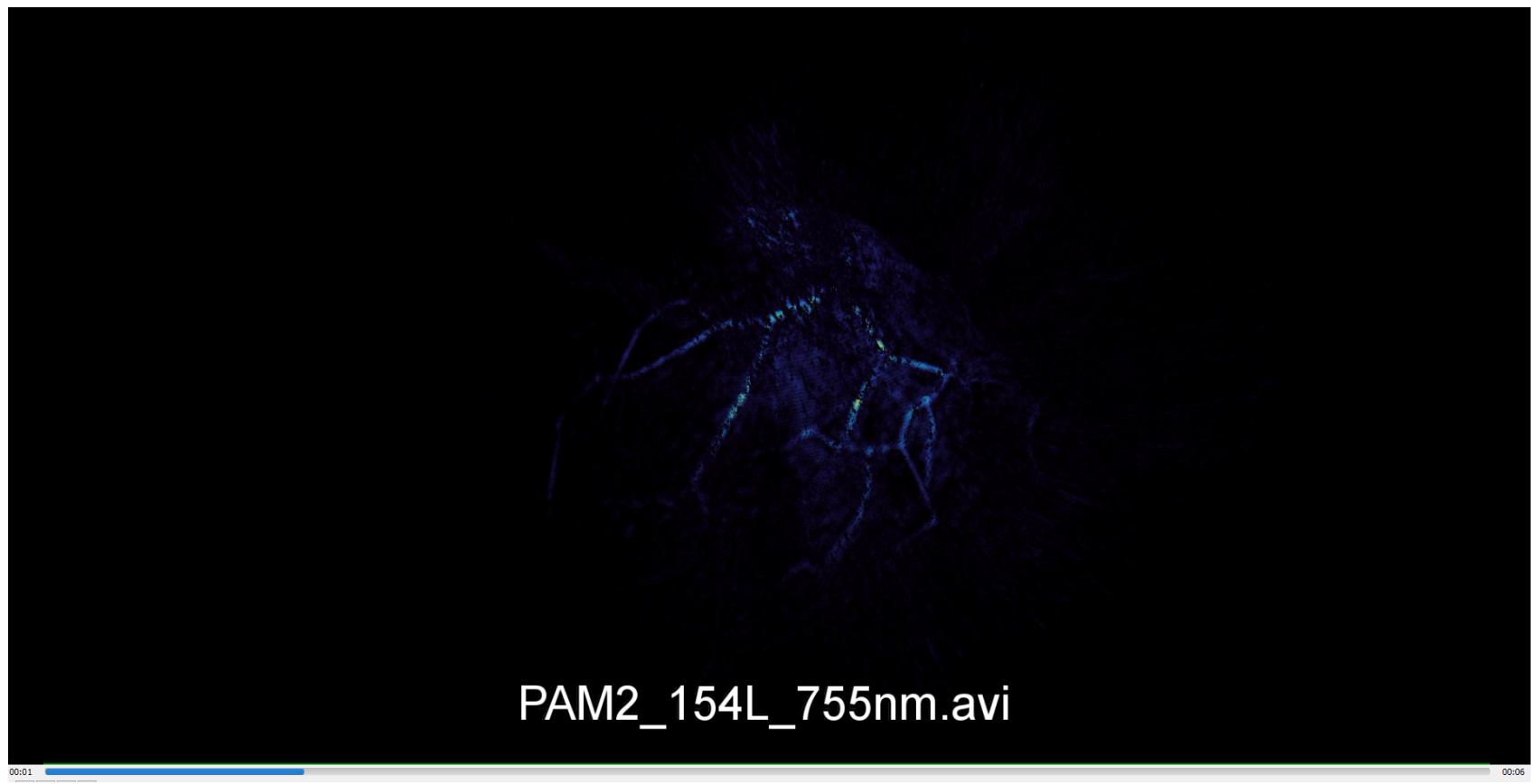

Video 1. Screenshot of video of rotating reconstructed 3D volume of the right breast of healthy volunteer \#154 (21 projection angles, 100 laser pulses per average, excitation with $755 \mathrm{~nm}$ ). File can be found at http://dx.doi.org/10.1117/12.2507448.1 


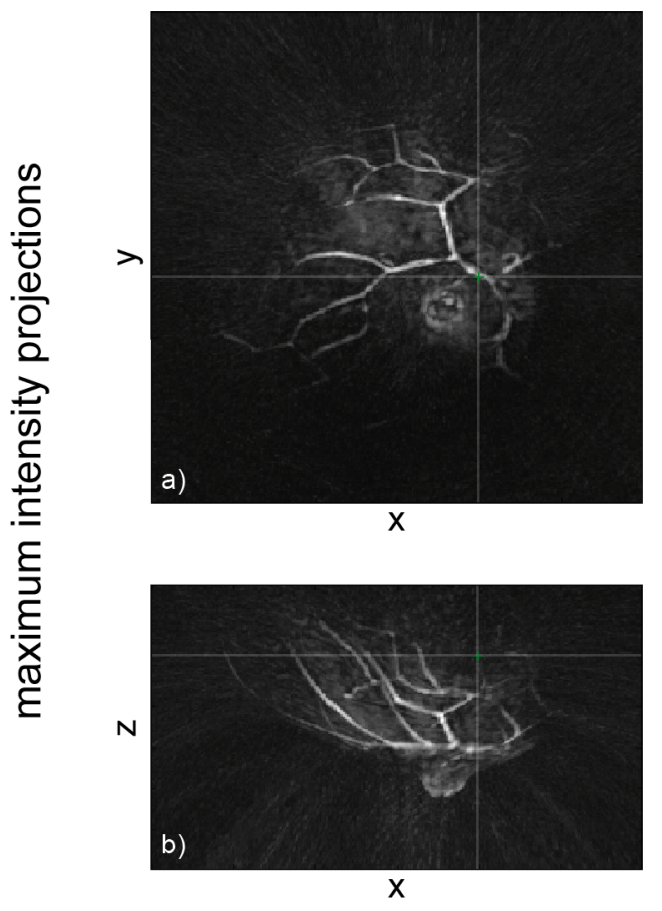

$20 \mathrm{~mm}$
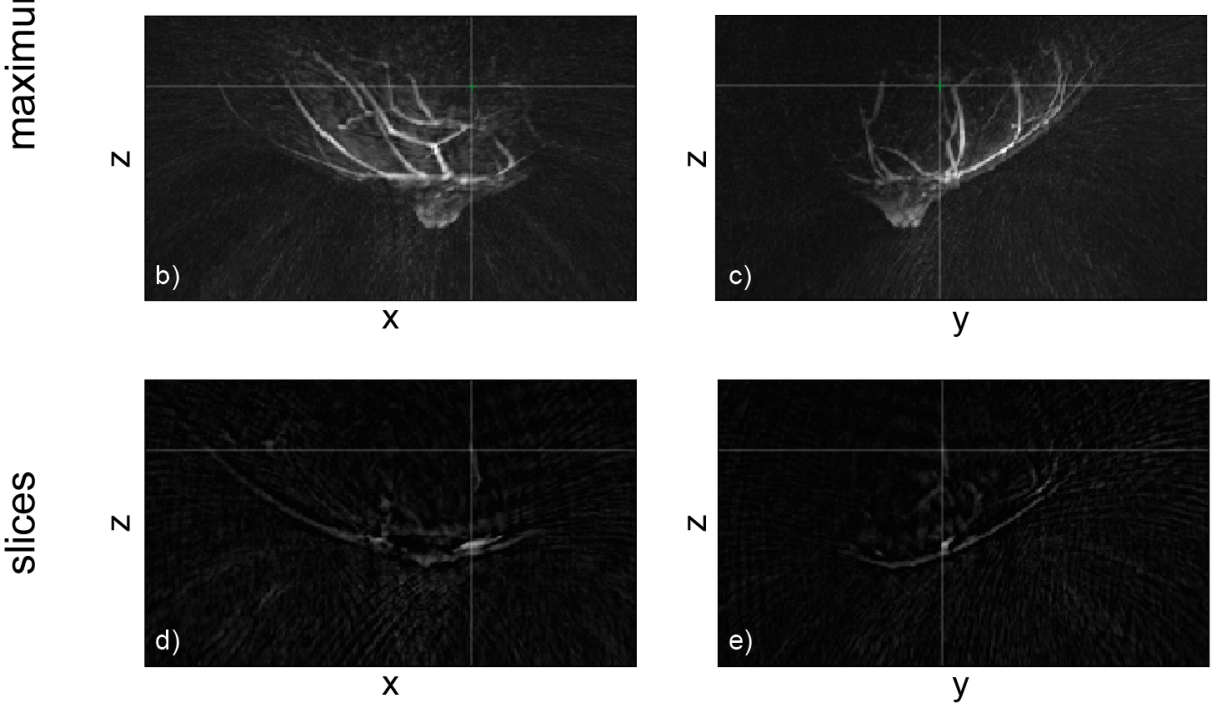

Figure 3. Reconstructed MIPs of the left breast of healthy volunteer \#154. Lines indicate the coordinates where slices (with thickness $=1.5 \mathrm{~mm}$ ) were obtained from. a) Coronal (XY view). b) Sagittal (XZ) view). c) Transverse (YZ) view. d) Sagittal (XZ) slice. e) Transverse (YZ) slice.

\section{DISCUSSION \& CONCLUSION}

The Twente Photoacoustic Mammoscope 2 (PAM 2) has been developed to acquire high quality images of the breast. The contrast and detail of blood vessels in the images is highly promising. The images presented here reveal blood vessels down to approximately $20 \mathrm{~mm}$. Nipple and breast contour are distinguishable both in projected views (MIPs and LMIPs) as well as a video (Video 1) where the reconstructed volume is rotating.

So far, detailed images of vascularization in healthy breasts have been obtained with PAM 2. Given the altered vascularization in and around breast tumors ${ }^{19}$, ${ }^{20}$ we expect that a photoacoustic image of (a breast with) a tumor will reveal abnormalities compared to that of a healthy breast. In future we will report on breast images acquired from highly suspect breasts.

Here we showed images obtained with an illumination wavelength of $755 \mathrm{~nm}$. A comparison of images obtained with $755 \mathrm{~nm}$ and $1064 \mathrm{~nm}$ show a good overlap of visualization of structures (not shown here, to be reported in a future publication), opening up possibilities for a functional analysis of the relative contributions of oxy- and deoxy- 
hemoglobin to the absorption contrast. This is a clinically relevant parameter in assessing malignancy of breast tumors ${ }^{21}$, 22 .

As mentioned, the system in its current form is not always capable of imaging the full breast. Firstly, due to the fact that the breast is hanging freely inside the imaging tank, it is possible to misalign it. This results in images where a portion of the breast is not visualized (for example the absence of the caudal part of the breast in Figure 2c). Therefore, we are working on a way to immobilize the breast during a measurement. This will position the breast inside the imaging tank and will reduce the effects of motion artifacts (observed in other measurements). Secondly, due to limited depth visualization, the inside of the breast is not visualized. Efforts are on to implement more sophisticated image reconstruction algorithms based on iterative methods ${ }^{23}$. We are optimistic that the PAM 2 system, with the intended improvements, has a good chance of visualizing abnormalities in the female breast.

\section{ACKNOWLEDGEMENTS}

Authors acknowledge Stichting Achmea Gezondheidszorg for funding in project Z620 and the Life Sciences \& Health LSH-TKI PPP project SACAMIR (LSHM17007) within the Health Holland Call. We acknowledge the European Union's Horizon 2020 Research and Innovation Action, H2020 ICT 2016-2017, an initiative of the Photonics 21 Public Private Partnership, for the project PAMMOTH under grant agreement No 732411.

\section{REFERENCES}

[1] Torre, L. A., Bray, F., Siegel, R. L., Ferlay, J., Lortet-Tieulent, J. and Jemal, A., "Global cancer statistics, 2012", CA Cancer Journal for Clinicians, 65(2), 87-108 (2015).

[2] Baltzer, P. A. T., Kapetas, P., Marino, M. A. and Clauser, P., "New diagnostic tools for breast cancer", Memo Magazine of European Medical Oncology, 10(3), 175-180 (2017).

[3] Diot, G., Metz, S., Noske, A., Liapis, E., Schroeder, B., Ovsepian, S. V., Meier, R., Rummeny, E. and Ntziachristos, V., "Multispectral Optoacoustic Tomography (MSOT) of human breast cancer", Clinical Cancer Research, 23(22), 6912-6922 (2017).

[4] Becker, A., Masthoff, M., Claussen, J., Ford, S. J., Roll, W., Burg, M., Barth, P. J., Heindel, W., Schäfers, M., Eisenblätter, M. and Wildgruber, M., "Multispectral optoacoustic tomography of the human breast: characterisation of healthy tissue and malignant lesions using a hybrid ultrasound-optoacoustic approach", European Radiology, 28(2), 602-609 (2018).

[5] Oraevsky, A. A., Clingman, B., Zalev, J., Stavros, A. T., Yang, W. T. and Parikh, J. R., "Clinical optoacoustic imaging combined with ultrasound for coregistered functional and anatomical mapping of breast tumors", Photoacoustics, 12, 30-45 (2018).

[6] Menezes, G. L. G., Pijnappel, R. M., Meeuwis, C., Bisschops, R., Veltman, J., Lavin, P. T., Van De Vijver, M. J. and Mann, R. M., "Downgrading of breast masses suspicious for cancer by using optoacoustic breast imaging", Radiology, 288(2), 355-365 (2018).

[7] Neuschler, E. I., Butler, R., Young, C. A., Barke, L. D., Bertrand, M. L., Böhm-Vélez, M., Destounis, S., Donlan, P., Grobmyer, S. R., Katzen, J., Kist, K. A., Lavin, P. T., Makariou, E. V., Parris, T. M., Schilling, K. J., Tucker, F. L. and Dogan, B. E., "A pivotal study of optoacoustic imaging to diagnose benign and malignant breast masses: A new evaluation tool for radiologists", Radiology, 287(2), 398-412 (2018).

[8] Toi, M., Asao, Y., Matsumoto, Y., Sekiguchi, H., Yoshikawa, A., Takada, M., Kataoka, M., Endo, T., Kawaguchi-Sakita, N., Kawashima, M., Fakhrejahani, E., Kanao, S., Yamaga, I., Nakayama, Y., Tokiwa, M., Torii, M., Yagi, T., Sakurai, T., Togashi, K. and Shiina, T., "Visualization of tumor-related blood vessels in human breast by photoacoustic imaging system with a hemispherical detector array", Scientific Reports, 7 , 41970 (2017).

[9] Matsumoto, Y., Asao, Y., Sekiguchi, H., Yoshikawa, A., Ishii, T., Nagae, K.-i., Kobayashi, S., Tsuge, I., Saito, S., Takada, M., Ishida, Y., Kataoka, M., Sakurai, T., Yagi, T., Kabashima, K., Suzuki, S., Togashi, K., Shiina, T. and Toi, M., "Visualising peripheral arterioles and venules through high-resolution and large-area photoacoustic imaging", Scientific Reports, 8(1), 14930 (2018). 
[10] Oraevsky, A. A., Su, R., Nguyen, H., Moore, J., Lou, Y., Bhadra, S., Forte, L., Anastasio, M. and Yang, W., "Full-view 3D imaging system for functional and anatomical screening of the breast". Proc. SPIE 10494, 104942Y (2018).

[11] Lin, L., Hu, P., Shi, J., Appleton, C. M., Maslov, K., Li, L., Zhang, R. and Wang, L. V., "Single-breath-hold photoacoustic computed tomography of the breast", Nature Communications, 9(1), 2352 (2018).

[12] Manohar, S., Kharine, A., Van Hespen, J. C. G., Steenbergen, W. and Van Leeuwen, T. G., "The Twente Photoacoustic Mammoscope: System overview and performance", Physics in Medicine and Biology, 50(11), 2543-2557 (2005).

[13] Manohar, S., Vaartjes, S. E., Van Hespen, J. C. G., Klaase, J. M., Van Den Engh, F. M., Steenbergen, W. and Van Leeuwen, T. G., "Initial results of in vivo non-invasive cancer imaging in the human breast using nearinfrared photoacoustics", Optics Express, 15(19), 12277-12285 (2007).

[14] Heijblom, M., Piras, D., Xia, W., Van Hespen, J. C. G., Klaase, J. M., Van Den Engh, F. M., Van Leeuwen, T. G., Steenbergen, W. and Manohar, S., "Visualizing breast cancer using the Twente photoacoustic mammoscope: What do we learn from twelve new patient measurements?", Optics Express, 20(11), 1158211597 (2012).

[15] Heijblom, M., Piras, D., Brinkhuis, M., van Hespen, J. C. G., van den Engh, F. M., van der Schaaf, M., Klaase, J. M., van Leeuwen, T. G., Steenbergen, W. and Manohar, S., "Photoacoustic image patterns of breast carcinoma and comparisons with Magnetic Resonance Imaging and vascular stained histopathology", Scientific Reports, 5, 11778 (2015).

[16] Heijblom, M., Piras, D., van den Engh, F. M., van der Schaaf, M., Klaase, J. M., Steenbergen, W. and Manohar, S., "The state of the art in breast imaging using the Twente Photoacoustic Mammoscope: results from 31 measurements on malignancies", European Radiology, 26(11), 3874-3887 (2016).

[17] Haltmeier, M., Schuster, T. and Scherzer, O., "Filtered backprojection for thermoacoustic computed tomography in spherical geometry", Mathematical Methods in the Applied Sciences, 28(16), 1919-1937 (2005).

[18] Sato, Y., Shiraga, N., Nakajima, S., Tamura, S. and Kikinis, R., "Local maximum intensity projection (LMIP): A new rendering method for vascular visualization”, Journal of Computer Assisted Tomography, 22(6), 912917 (1998).

[19] Hanahan, D. and Weinberg, R. A., "Hallmarks of Cancer: The Next Generation", Cell, 144(5), 646-674 (2011).

[20] Siemann, D. W., "The unique characteristics of tumor vasculature and preclinical evidence for its selective disruption by Tumor-Vascular Disrupting Agents", Cancer Treatment Reviews, 37(1), 63-74 (2011).

[21] Höckel, M. and Vaupel, P., "Tumor hypoxia: Definitions and current clinical, biologic, and molecular aspects", Journal of the National Cancer Institute, 93(4), 266-276 (2001).

[22] Ntziachristos, V., Yodh, A. G., Schnall, M. D. and Chance, B., "MRI-Guided Diffuse Optical Spectroscopy of Malignant and Benign Breast Lesions", Neoplasia, 4(4), 347-354 (2002).

[23] Boink, Y. E., Lagerwerf, M. J., Steenbergen, W., Van Gils, S. A., Manohar, S. and Brune, C., “A framework for directional and higher-order reconstruction in photoacoustic tomography”, Physics in Medicine and Biology,

63(4), 045018 (2018). 